\title{
RESISTÊNCIA À COMPRESSÃO MECÂNICA DE COM- PÓSITOS DE POLIURETANO E CIMENTO, COM ADIÇÃO DE LODO DE VIDRO COMO SUBSTITUTO DA AREIA
}

\author{
RESISTANCE TO MECHANICAL COMPRESSION OF POLYURETHANE AND CEMENT COMPOSITES, \\ WITH ADDITION OF GLASS SLUDGE AS A SAND SUBSTITUTE
}

POLYANA BAUNGARTEN | UNISUL

RACHEL FAVERZANI MAGNAGO, Dra.| UNISUL

\begin{abstract}
RESUMO
As placas de poliuretano (PU) são utilizadas como isolante térmico e acústico devido ao seu ótimo desempenho, no entanto não apresentam compatibilidade com cimento, limitando o uso destas na construção civil. Outra necessidade urgente é de encontrar fins para resíduos industriais, tais como lodo de vidro, podendo ser através da sua incorporação em novos materiais. O objetivo deste estudo foi preparar compósitos de poliuretano e cimento, com incorporação de lodo da lapidação de vidro e insumo de areia, com o intuito de investigar o comportamento da resistência mecânica em relação ao tempo, a densidade aparente dos espécimes e a influência da cura ao ar e cura em água. Para tanto foi realizado a preparação dos compósitos pela reação de policondensação de polipropilenoglicol e tolueno 2,6-diisocianato (2,4-TDI/2,6TDI, 80/20) e adição de cimento e areia/resíduo de vidro. O teste de resistência mecânica de compressão com 35 dias teve como melhor resultado o espécime Pu_Cim, porém foi constatada uma redução de 39,93\% de sua resistência no teste de 90 dias, sendo ultrapassado pelos espécimes Pu_Cim_Areia e Pu_Cim_Vidro, que ao contrário do Pu_Cim, tiveram um expressivo aumento na sua resistência de 10,33 e 77,69\%, respectivamente. Observou-se também que o período de cura em água foi essencial para o aumento da resistência do espécime Pu_Cim e que o espécime com lodo de vidro foi menos denso entre os três. Em suma, o espécime Pu_Cim_Vidro apresentou melhores propriedades mecânicas alavancando os benefícios da substituição de parte do cimento pelo lodo de vidro, sendo ecologicamente e economicamente viável.
\end{abstract}

PALAVRAS-CHAVE: Poliuretano; Resíduo Industrial; Resistência mecânica.

\begin{abstract}
Polyurethane (PU) sheets are used as thermal and acoustic insulation due to their excellent performance, however they do not have compatibility with cement, limiting their use in civil construction. Another urgent need is to find ends for industrial waste, such as glass sludge, which may be through its incorporation into new materials. The objective of this study was to prepare polyurethane and cement composites, with the incorporation of glass-cutting sludge and sand input, in order to investigate the behavior of mechanical resistance over time, the apparent density of specimens and the influence of curing. air and cure in water. For this purpose, the preparation of the composites was carried out by the polycondensation reaction of polypropylene glycol and toluene 2,6-diisocyanate (2,4TDI / 2,6-TDI, 80/20) and the addition of cement and sand / glass residue. The 35-day mechanical compressive strength test had the best result for the Pu_Cim specimen, but it was found a 39.93\% reduction in its resistance in the 90-day test, being surpassed by the Pu_Cim_AreiaandPu_Cim_Vidrospecimens, which unlikePu_Cim, had a significant increase in theirresistance of 10.33 and $77.69 \%$, respectively. It was also observed that the curing period in water was essential for increasing the strength of the Pu_Cim specimen and that the specimen with glass sludge was less dense among the three.In short, thePu_Cim_Vidro specimen showed bettermechanical properties, leveraging the benefits of replacing part of the cement with glass sludge, being ecologically and economically viable.
\end{abstract}

KEY WORDS: Poliuretano; Resíduo Industrial; Resistência mecânica. 


\section{INTRODUÇÃO}

A construção civil e todos seus produtos e processos são considerados um dos maiores geradores de resíduos no mundo, acarretando um crescente interesse no reaproveitamento destes resíduos. Uma possibilidade é incorporá-los através de inovação e tecnologia como matéria prima para confecção de novos produtos, atribuindo valor e um ciclo de vida maior para os mais variados tipos de resíduos (CARVALHO et al., 2015; DEMIREL, 2013). Além disso, a necessidade de encontrar fins para resíduos da indústria local que ofereçam conforto, segurança e sustentabilidade ainda é um forte estímulo para pesquisas e desenvolvimento de produtos que incorporem resíduos (ESTRELLA, 1996).

Os materiais de isolamento térmico exercem um papel importante quanto ao conforto térmico e seu uso contribui efetivamente para reduzir a energia necessária para aquecimento ou refrigeração de ambientes gerando maior eficiência energética para manter uma boa temperatura interna (MAZOR et al., 2011).

O poliuretano é um polímero termoplástico utilizado na construção civil, devido ao seu excelente desempenho como isolante térmico e acústico. Além disso, pode adquirir qualquer formato, é leve e de fácil manuseio (GUO et al., 2015; MEIRELLES, 2014; VLADIMIROV et al., 2011). Porém, apesar de seus benefícios e durabilidade no médio e longo prazo, ainda é visto como um produto caro, quando comparado com seus concorrentes como, por exemplo, o isopor (EPS).

O lodo de vidro é um resíduo gerado pela fabricação, tratamento, perfuração, corte e manuseio de peças de vidro. A capacidade nominal de produção de vidro plano no Brasil foi de 7530 toneladas / dia em 2019 (ABRAVIDRO, 2019), como consequência este resíduo representa, em média 3\% de todos os resíduos urbanos no brasil e infelizmente menos de $50 \%$ dele foi reciclado; o restante é descartado diretamente em aterros sanitários (CEMPRE, 2017). Esse resíduo pode ser utilizado na remanufatura de vidro, caso ele esteja na forma de cacos, porque são inertes e não biodegradáveis. No entanto, as micropartículas de vidro do polimento e da perfuração são um risco ambiental, porque sua reciclagem não é economicamente viável.

A partir disso, vem sendo feitos diferentes estudos de aplicações para o lodo de vidro. A Tabela 1 demonstra artigos com esse tema publicados entre os anos de 2015 a 2020, bem como as revistas onde foram publicados.

\begin{tabular}{|l|l|l|}
\hline Artigo & Revista & Aplicação \\
\hline Kim, Yi e & $\begin{array}{l}\text { Construction and } \\
\text { Zi, (2015). }\end{array}$ & $\begin{array}{l}\text { Substituição parcial do } \\
\text { cimento por lodo de } \\
\text { vidro na argamassa. }\end{array}$ \\
\hline
\end{tabular}

\begin{tabular}{|l|l|l|}
\hline $\begin{array}{l}\text { Nandi et } \\
\text { al., (2015). }\end{array}$ & $\begin{array}{l}\text { Journal of Cleaner } \\
\text { Production }\end{array}$ & $\begin{array}{l}\text { Engobes de cerâmica a partir } \\
\text { do lodo de cerâmica e vidro. }\end{array}$ \\
\hline $\begin{array}{l}\text { Kazmi et } \\
\text { al., (2018) }\end{array}$ & $\begin{array}{l}\text { Journal of Cleaner } \\
\text { Production }\end{array}$ & $\begin{array}{l}\text { Tijolos de argila queimada } \\
\text { usando lodo de vidro. }\end{array}$ \\
\hline $\begin{array}{l}\text { ALVARENGA } \\
\text { et al., (2019) }\end{array}$ & $\begin{array}{l}\text { Materiales de } \\
\text { Construcción }\end{array}$ & $\begin{array}{l}\text { Substituição parcial do } \\
\text { cimento por micropar- } \\
\text { tículas de vidro com } \\
\text { resíduo de soda e cal. }\end{array}$ \\
\hline $\begin{array}{l}\text { COPPOLA et } \\
\text { al., (2019) }\end{array}$ & $\begin{array}{l}\text { Journal of the } \\
\text { Society }\end{array}$ & $\begin{array}{l}\text { Ativação alcalina de lodo } \\
\text { de mármore com adição } \\
\text { de resíduos de vidro. }\end{array}$ \\
\hline $\begin{array}{l}\text { SHOAEl et } \\
\text { al., (2020) }\end{array}$ & $\begin{array}{l}\text { Construction and } \\
\text { Building Materials }\end{array}$ & $\begin{array}{l}\text { Incorporação de pó de vidro } \\
\text { como percursor parcial } \\
\text { de cimento Portland. }\end{array}$ \\
\hline
\end{tabular}

Tabela 1 - Aplicações do lodo de vidro apresentadas em diferentes estudos. Fonte: Autores

Segundo levantamento apresentado na Tabela 1, entre os anos de 2015 a 2020, o lodo de vidro foi estudado em diferentes aplicações, muitas vezes com o intuito de substituir parcialmente o cimento. A combinação cimento/lodo de vidro apresenta boa reatividade pozolânica e gera um aumento de resistência mecânica (KAZMl et al., 2018; SHOAEI et al., 2020). Nesta pesquisa, buscou-se a combinação das características desses materiais com uma matriz de poliuretano com o objetivo de criar uma placa termo acústica com propriedades aprimoradas, mais sustentável e econômica.

O cimento foi incorporado a todos os espécimes principalmente para aumentar resistência mecânica de compressão e possibilidade de acabamento cimentício. O lodo de vidro foi selecionado por ser um resíduo, um material incombustível, isolante térmico, inerte, de baixo custo e de alta durabilidade química (LUZ; RIBEIRO, 2008; VARGAS; WIEBECK, 2007; BOYD et al., 1994).

A areia por sua vez, foi utilizada para comparação com os compostos com vidro, principalmente devido à similaridade na sua composição em relação ao lodo de vidro. $O$ lodo de vidro apresenta em sua composição, em média, $68 \%$ em massa de $\mathrm{SiO} 2,10 \%$ de $\mathrm{CaO}, 3 \%$ de $\mathrm{Al} 2 \mathrm{O} 3,0,35 \%$ de $\mathrm{Fe} 2 \mathrm{O} 3$, além de outros compostos em menores quantidades (GUIGNONE, 2017). A sílica (SiO2) é o principal componente da areia e a principal matéria prima para o vidro. Em vista dessa correlação, gerou-se um interesse em conhecer e comparar a influência da incorporação desse insumo.

Deste modo, foram avaliadas a influência da cura em ar e cura em água/ar em espécimes de poliuretano e cimento, além da investigação da resistência mecânica à compressão com o passar do tempo para espécimes de poliuretano e cimento com a incorporação de lodo de vidro ou areia. Deste modo, o desenvolvimento de produtos com substituição de um insumo por um resíduo, em virtude da urgente necessidade de limitar o uso de recursos naturais. 


\section{PROCEDIMENTO EXPERIMENTAL}

Foram adquiridos o lodo da lapidação de vidro (doação de Personal Glass), areia (Guarezi), cimento Portland (CPV-ARI, Itambé) e os reagentes polipropilenoglicol (AudazBrasil) e tolueno-2,6-diisocianato (2,4-TDI/2,6-TDI, 80/20, Audaz Brasil).

O lodo de vidro foi lavado e filtrado utilizando funil de Buchner e bomba a vácuo, seco a $70^{\circ} \mathrm{C}$ em estufa por 24 h e tamisado em peneiras de inox com abertura de malha de $45 \mu \mathrm{m}$. A areia foi tamisada em uma peneira de inox com abertura de malha de $150 \mu \mathrm{m}$. Logo após o preparo dos materiais, esses foram dosados, homogeneizados e inseridos em moldes de madeira revestidos com fita de

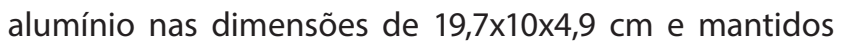
por cerca de 1 hora. Após desmoldados cada espécime foi cortado em corpos de prova nas medidas $5 \times 5 \times 4 \mathrm{~cm}$ para a realização do teste de resistência mecânica. No total, foram confeccionados 8 espécimes, gerando 40 corpos de prova, pela reação de policondensação do poliolpoliéter e toluenodiisocianato com cimento e incorporação de areia ou lodo de vidro.

O primeiro ensaio consistiu em avaliar a resistência mecânica a compressão de espécimes com a cura submerso em água/ar e a cura ao ar. Para o estudo da cura submersa em água foram preparados 10 corpos de prova de poliuretano com cimento (Pu_Cim), 5 deles permaneceram 7 primeiros dias submersos em água e mais 28 dias curando ao ar. $O$ segundo grupo permaneceu 35 dias de cura ao ar.

Para o estudo de resistência mecânica de compressão em relação ao tempo de cura foram preparados 6 grupos de 5 corpos de prova nas composições poliuretano com cimento (Pu_Cim), poliuretano com cimento e areia $\left(\mathrm{Pu}_{-}\right.$ Cim_Areia) e poliuretano com cimento e lodo de vidro (Pu_Cim_vidro). O tempo de cura seguiu dois protocolos, (1) 7 dias de cura submersa em água e 28 dias de cura ao ar (total 35 dias de cura) e (2) 7 dias de cura submersa em água e 83 dias de cura ao ar (total 90 dias de cura).

Na Tabela 2 tem-se o número de espécimes preparados, e a quantidade de reagentes usados para sua confecção, em gramas.

\begin{tabular}{|l|l|l|l|l|l|}
\hline & No & Poliol & Isocianato & Cimento & Carga \\
\hline Pu_Cim & 4 & 48,9 & 73,6 & 73,6 & - \\
\hline $\begin{array}{l}\text { Pu_Cim_ } \\
\text { Vidro }\end{array}$ & 2 & 48,9 & 73,6 & 46,3 & 27,3 \\
\hline $\begin{array}{l}\text { Pu_ } \\
\text { Cim } \\
\text { Areia }\end{array}$ & 2 & 48,9 & 73,6 & 46,3 & 27,3 \\
\hline
\end{tabular}

Tabela 2 - Quantidades dos reagentes em massa ( $\mathrm{g}$ ) usados para confecção dos espécimes. Fonte: Autores
Os ensaios de resistência mecânica foram realizados no equipamento EMIC modelo DL 30000, célula de carga de $5 \mathrm{kN}$. Submeteu-se então, os corpos de prova à incrementos de pressão até deformação plástica (ASTM E2954).

Para o ensaio de densidade aparente, utilizou-se a balança analítica para obter a massa dos materiais e calculou-se o volume através das medidas com um paquímetro, obtendo o valor da densidade aparente e desvio padrão (ASTM D1622/D1622M).

\section{RESULTADOS E DISCUSSÕES}

Os espécimes poliuretano e cimento ( $\left.\mathrm{Pu} \_\mathrm{Cim}\right)$, poliuretano, cimento e areia (Pu_Cim_Areia) e poliuretano, cimento e lodo de vidro (Pu_Cim_Vidro) desenvolvidos estão apresentados na Figura 1.

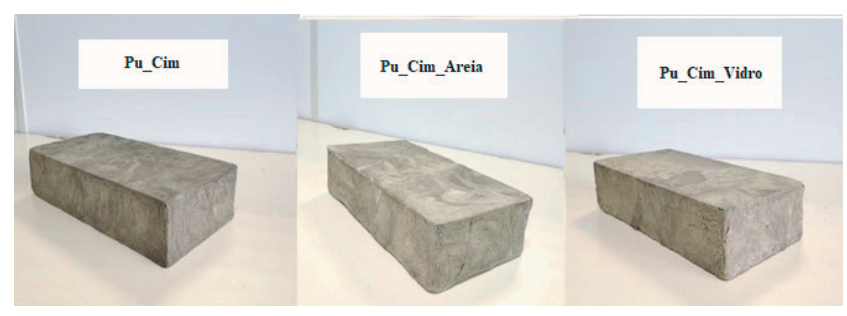

Figura 1 - Imagens dos espécimes de poliuretano com cimento (Pu_Cim), poliuretano com cimento e areia ( $\mathrm{Pu} \_\mathrm{Cim} \_$Areia) e poliuretano com cimento e lodo de vidro ( $\mathrm{Pu}$ _Cim_vidro). Fonte: Autores

Visualmente, na Figura 1, os espécimes preparados apresentaram aparência semelhante, superfície uniforme e bom aspecto visual, não demostrando deformação ou esfarelamento.

Na Figura 2 tem-se os resultados de resistência mecânica de compressão para os espécimes de Pu_Cim com cura ao ar e com cura em água/ar.

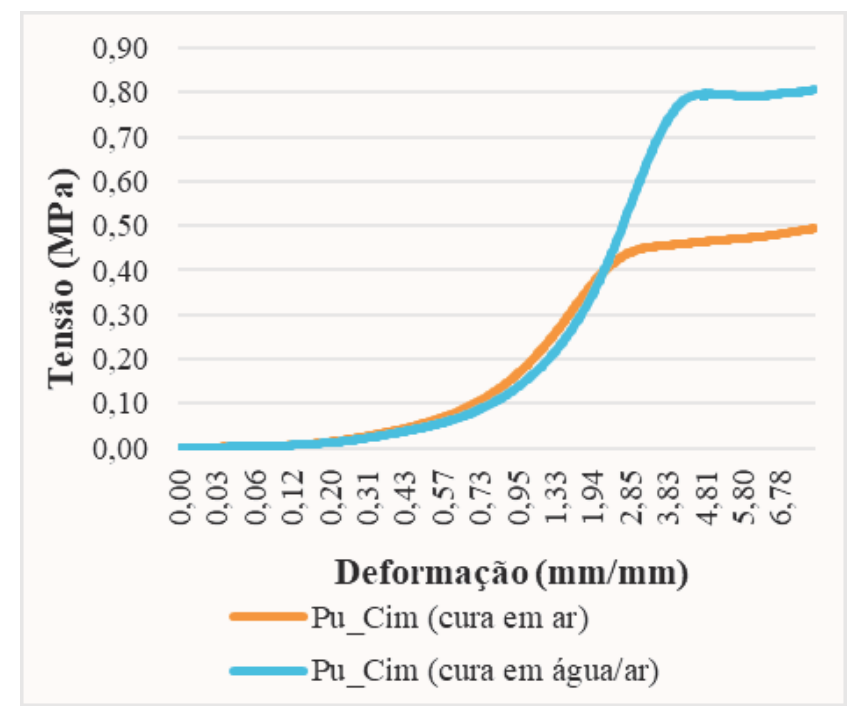

Figura 2 - Resistência mecânica de compressão para Pu_Cim (cura emágua/ar) e Pu_Cim (cura em ar). Fonte: Autores 
A Figura 2 foi gerada a partir ensaio de compressão, os resultados obtidos consistem na relação entre a deformação linear, obtida pela medida da distância entre as placas que comprimem o corpo de prova, em função da carga de compressão aplicada em cada instante. Estes valores de força são divididos pela área inicial do espécime a fim de obter a tensão.

Os espécimes de Pu_Cim apresentaram, conforme Figura 1, comportamento elástico plástico independentemente do processo de cura, deste modo atribuiu-se esse comportamento à matriz de PU (MARQUES et al., 2018). $O$ espécime com cura nos 7 primeiros dias submersos em água e mais 28 dias ao ar apresentou resistência máxima de compressão superior, com um valor de $0,7914 \mathrm{MPa}$, enquanto que o espécime com cura de 35 dias ao ar obteve $0,4418 \mathrm{MPa}$, o que faz da cura submersa em água uma etapa fundamental para a conquista de resistência mecânica a compressão do espécime Pu_Cim.

A cura em água é fundamental devido a reação entre o cimento com a água. Essa reação provoca a hidratação dos silicatos e aluminatos formando silicato de cálcio hidratado [Tobermita], hidróxido de cálcio [Portlandita: $\mathrm{Ca}(\mathrm{OH})_{2}$ ] e sulfoaluminatos de cálcio hidratados [Etringita]. O surgimento destas fases hidratadas ocorre em diferentes velocidades, o que confere características importantes como o enrijecimento, fornecendo resistência ao cimento (COUTINHO, 1997; PETRUCCI, 1998; ISAIA, 2005; MEHTA; MONTEIRO, 2008; NEVILLE; BROOKS, 2012).

Por sua vez, a cura em ar gera uma reação entre o cimento hidratado e dióxido de carbono, conhecida como carbonatação. Na presença de umidade, o $\mathrm{CO}_{2}$ que está presente na atmosfera forma ácido carbônico, que reage com o $\mathrm{Ca}(\mathrm{OH})_{2}$ formando $\mathrm{CaCO}_{3}$ (NEVILLE; BROOKS, 2012). A carbonatação ocorre da superfície para o interior, é extremamente lenta e traz benefícios como aumento da resistência e durabilidade, a partir da redução da porosidade da matriz pela precipitação dos cristais de $\mathrm{CaCO}_{3}$ nos poros (YUAN et al., 2013; MO, 2013; ROSTAMl et al., 2012; NEVILLE; BROOKS, 2012).

$\mathrm{Na}$ Figura 3 tem-se os resultados do ensaio de densidade aparente para os espécimes Pu_Cim, Pu_Cim_Areia, Pu_Cim_Vidro, com 35 e 90 dias.

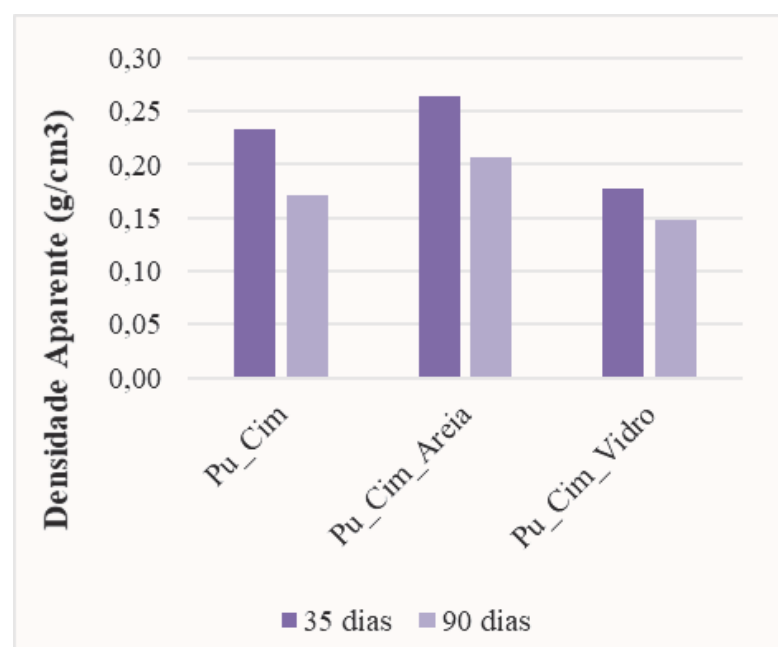

Figura 3 - Densidade aparente para os espécimes Pu_Cim, Pu_Cim_Areia e Pu_Cim_Vidro. Fonte: Autores

Observou-se na Figura 3, que todos os corpos de prova tiveram uma redução na densidade de 35 para 90 dias de cura. Isso aconteceu porque os espécimes passaram seus 7 primeiros dias submersos em água e naturalmente perderam parte dessa água com o passar do tempo.

O material mais denso foi o Pu_Cim_Areia com 0,2632 g/ $\mathrm{cm} 3$ em 35 dias e $0,2071 \mathrm{~g} / \mathrm{cm} 3 \mathrm{em} 90$ dias, seguido do Pu_ Cim com 0,2333 g/cm3 para 35 dias e 0,1709 g/cm3 para 90 dias e por último Pu_Cim_Vidro com 0,1776 g/cm3 para 35 dias e $0,1478 \mathrm{~g} / \mathrm{cm} 3$ para 90 dias. Esta diferença deve-se provavelmente a capacidade de reagir com a água que varia de acordo com a adição dos diferentes materiais ao poliuretano.

A Figura 4 apresenta os resultados do teste de resistência mecânica de compressão para espécimes Pu_Cim, Pu_Cim_Areia e Pu_Cim_Vidro com 35 dias de cura.

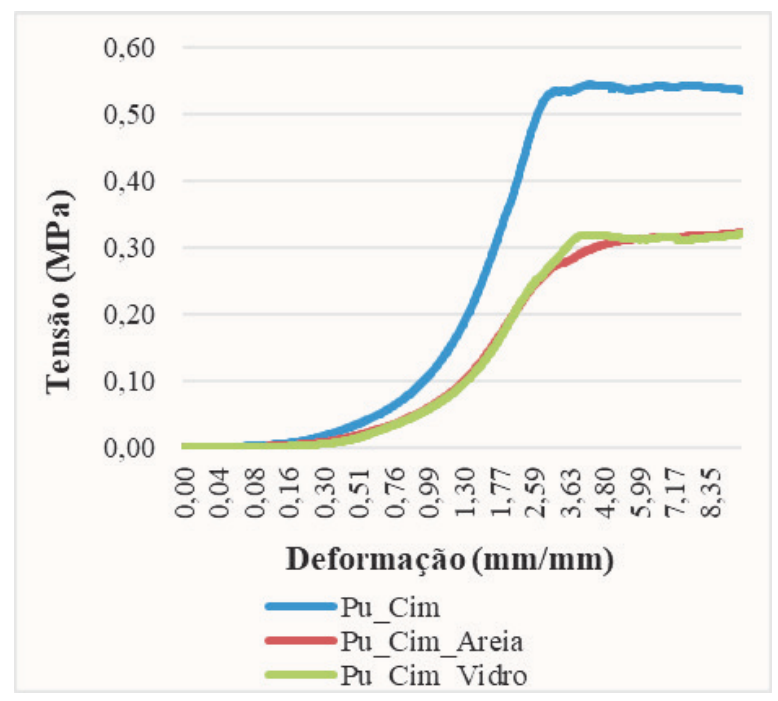

Figura 4 - Resistência mecânica de compressão com 35 dias de cura para Pu_Cim, Pu_Cim_ Areia e Pu_Cim_Vidro.

Fonte: Autores 
O corpo de prova de Pu_Cim atingiu uma resistência de 0,5069 MPa enquanto que os corpos de prova de Pu_Cim_Vidro e Pu_Cim_Areia obtiveram 0,3098 e 0,2765 $\mathrm{MPa}$, respectivamente. A partir disso, observa-se que a redução de cerca de $37 \%$ de cimento causou uma diminuição na resistência a compressão para os corpos de prova de PU_Cim_Areia e Pu_Cim_Vidro do material, entretanto, de acordo com a ASTM D1621-16, todos os espécimes revelaram resistência satisfatória para aplicação, pois estes não possuem função estruturante.

Constatou-se também na Figura 4 a similaridade nos resultados entre Pu_Cim_Areia e Pu_Cim_Vidro, o que foi coerente visto que possuem composição química similar.

A Figura 5 apresenta os resultados do teste de resistência mecânica de compressão para Pu_Cim, Pu_Cim_ Areia e Pu_Cim_Vidro com 90 dias de cura.

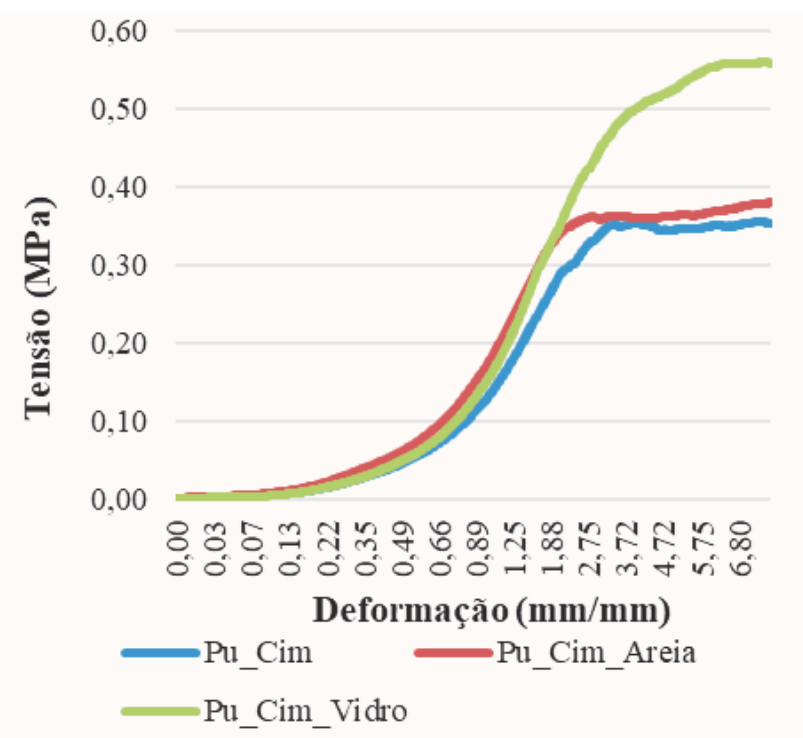

Figura 5 - Resistência mecânica de compressão com 90 dias de cura para Pu_Cim, Pu_Cim_ Areia e Pu_Cim_Vidro.

Fonte: Autores

A partir deste ensaio ficou evidente a influência do tempo de cura na resistência mecânica dos espécimes. O espécime Pu_Cim obteve 0,3033MPa, uma redução de 39,93\% se comparado ao teste de 35 dias. Isso pode ter acontecido devido a formação de porosidades ou interfaces de baixa resistência entre poliuretano e a matriz cimentícia, estas porosidades tornam o espécime vulnerável aos agentes agressivos do meio que, com o passar do tempo, causam a deterioração, reduzindo sua resistência e durabilidade (SIQUEIRA, 2004). Além disso, o cimento pode ter sofrido refração por secagem, neste fenômeno ocorre uma contração do cimento devido a perda da água, gerando fissuras e comprometendo sua resistência (NEVILLE; BROOKS, 2012).
Por outro lado, o espécime com areia teve um aumento de $10,33 \%$, atingindo $0,3418 \mathrm{MPa}$ e o espécime com lodo de vidro um expressivo aumento de $77,69 \%$, alcançando 0,4913MPa. Este aumento na resistência aconteceu possivelmente em razão do alto teor de sílica (SiO2) presente na areia e no lodo de vidro, que em contato com o hidróxido de cálcio reage formando uma quantidade extra de silicato de cálcio hidratado (GRAUPMANN et al., 2019; MYMRIN et al, 2017). Além disso, a adição do lodo de vidro pode reduzir significativamente o encolhimento por secagem de amostras curadas ao ar, porque opera como um agregado rígido (COPPOLA et al., 2019).

Mediante o exposto, nota-se que a substituição de parte do cimento por lodo de vidro gerou o espécime de melhor resultado no ensaio de resistência a compressão a longo prazo Pu_Cim_Vidro, uma alternativa ecológica que garante uma reutilização eficiente do resíduo de vidro, além de ter um custo mais acessível.

\section{CONCLUSÃO}

Espécimes de poliuretano com cimento, areia ou lodo de vidro foram preparados pela incorporação dos insumos inorgânicos aos reagentes poliol e isocianato. Para Pu_Cim foi observado maior resistência mecânica a compressão quando os espécimes foram curados submersos em água e ar. O melhor resultado no teste de resistência mecânica de compressão foi para Pu_Cim com 0,5069 MPa com 35 dias de cura em água e ar, enquanto que para Pu_Cim_Areia e Pu_Cim_Vidro foi semelhante a resistência mecânica a compressão, cerca de 0,3 MPa. Entretanto, o teste de resistência mecânica de compressão feito com 90 dias teve como melhor resultado o espécime com adição do lodo de vidro, com um acréscimo de $77,69 \%$ na sua resistência, ultrapassando o Pu_Cim, que com 90 dias de cura teve uma redução de $39,93 \%$ na sua resistência. $O$ espécime com areia obteve a menor variação de resistência com o passar do tempo, aumentou $10 \%$ que ainda sim pode ser considerado um valor significativo.

Em suma, com cura de 90 dias, sendo 7 dias submerso em água e seguido por cura ao ar, o espécime com melhor desempenho em relação a resistência mecânica de compressão foi o Pu_Cim_Vidro. Ele também foi o espécime mais apropriado economicamente devido ao baixo custo e ecologicamente devido ao reaproveitamento dos resíduos de vidro, que são em grande parte descartados incorretamente.

\section{REFERÊNCIAS}

ABRAVIDRO. Associação brasileira de distribuidores e processadores de vidros planos. Panorama Abravido. 
Brasília, 2018.

ALVARENGA, C.; HEIDERICK, O.; COUTO, T.; CETLIN, P.; SALES, R.; AGUILAR, M. T. P. Influence of soda-lime waste glass microparticles on workability and thermal properties of portland cement compounds. Materiales de Construcción, 2019.

AMERICAN SOCIETY FOR TESTING AND MATERIALS. Standard Test Method for Axial Compression Test of Reinforced Plastic and Polymer Matrix Composite Vertical Members, 2015.

AMERICAN SOCIETY FOR TESTING AND MATERIALS. Standard Test Method for Apparent Density of Rigid Cellular Plastics, 2014.

AMERICAN SOCIETY FOR TESTING AND MATERIALS. Standard Test Method for Compressive Properties of Rigid Cellular Plastics, 2016.

BATOOL, F.; BINDIGANAVILE, V.; Thermal conductivity of hydrated paste in cementbased foam microstructure, Adv. Civ. Eng. Mater. 7, 2018.

CEMPRE. Compromisso empresarial para reciclagem, 2017.

BOYD, D. C.; DANIELSON, P. S. \& THOMPSON, D.A. "Glass" in: Kirk-Othmer, Encyclopedia of Chemical Technology, vol.12, Ed. Wiley Interscience, USA, 1994.
COPPOLA, B.; PALMERO, P., MONTANARO, L.; TULLIANI, J.-M. Alkali-activation of marble sludge: Influence of curing conditions and waste glass addition. Journal of the European Ceramic Society, 2019

COUTINHO, A.S. Fabrico e propriedades do betão. Laboratório de engenharia civil, vol. I, Portugal, 1997. ESTRELLA, S. P. Diagnóstico de resíduos sólidos industriais em Santa Catarina - perspectivas de uso na construção civil. 193 f. Dissertação (Mestrado): Curso de Engenharia Civil, Universidade Federal de Santa Catarina, Florianópolis, 1996.

GRAUPMANN, O.; MARTINS, M. A.; CONSUL, M.; CECHIN, L. Análise da resistência à compressão de concretos com adição de cinza de aterro sanitário. Rio de Janeiro, vol. 24, 2019.

GUIGNONE, G. C. Desempenho de concretos com a utilização de resíduos da lapidação do vidro como substitutos parciais ao cimento. Dissertação (Mestrado em Engenharia Civil) - Vitória: Universidade Federal do Espírito Santo, 2017.

GUO, H., GAO, Q., OUYANG, C., ZHENG, K., \& XU, W. Research on properties of rigid polyurethane foam with heteroaromatic and brominated benzyl polyols. Journal of Applied Polymer Science, 2015.

ISAIA, G. C. Concreto ensino, pesquisa e realizações.
São Paulo: IBRACON, 2005.

KAZMI, S. M. S.; MUNIR, M. J.; WU, Y.; HANIF, A.; PATNAIKUNI, I. Thermal performance evaluation of eco-friendly bricks incorporating waste glass sludge. Journal of Cleaner Production. vol. 172, 2018.

$\mathrm{KIM}$, J.; YI, C.; ZI, G. Waste glass sludge as a partial cement replacement in mortar. Construction and Building Materials, 2015.

LEE. C.; WON, J.W.; JANG, W.; JUNG, W.; HAN, S. H.; KWAK, Y. H. Social conflict management framework for project viability: Case studies from Korean megaprojects, International Journal of Project Management, 2017.

LUZ, A.P.; RIBEIRO, S. Uso de pó de vidro como fundente para produção de grês porcelanato. Matéria vol. 13. Rio de Janeiro, 2008.

MARQUES, D. V.; BARCELOS, R. L.; SILVA, H. R. T.; EGERT, P.; PARMA, G. O. C.; GIROTTO, E.; CONSONI, D.; BENAVIDES, R.; SILVA, L.; MAGNAGO, R. F. Recycled Polyethylene Terephthalate-Based Boards for ThermalAcoustic Insulation. Journal Cleaner Production. vol. 189, 2018.

MAZOR, M.; MUTTO, J.; RUSSEL, D.; KEOLEIAN, G. Life cycle greenhouse gas emissions reduction from rigid thermal insulation use in buildings, Journal of Industrial Ecology 15 (2), 2011.

MEHTA, P. K.; MONTEIRO, P. J. M. Concreto. Microestrutura, propriedades e materiais. 3. ed. São Paulo: IBRACON, 2008.

MEIRELLES, S. Química verde: a indústria química e seus impactos na indústria da construção. Anais: Congresso Nacional de Excelência em Gestão. Rio de Janeiro, 2014.

MO, L., PANESAR, DAMAN, K. Accelerated carbonation - A potential approach to sequester CO2 in cement paste containing slag and reactive $\mathrm{MgO}$. Cement and Concrete Composites, vol. 43, 2013.

MYMRIN, V., STELLA, J. C., SCREMIM, C. B., PAN, R. C. Y., SANCHES, F. G.; ALEKSEEV, K.; PEDROSO, D. E.; MOLINETTI, A.; FORTINI, O. M. "Utilization of sediments dredged from marine ports as a principal component of composite material", Journal of Cleaner Production, vol. 142, 2017.

NANDI, V. S.; RAUPP-PEREIRA, F.; MONTEDO, O. R. K.; OLIVEIRA, A. P. N. The use of ceramic sludge and recycled glass to obtain engobes for manufacturing ceramic tiles. Journal of Cleaner Production, 2015. PETRUCCI, E. G. R. Concreto de cimento Portland. 13. ed. São Paulo: Globo, 1998. 
ROSTAMI V., SHAO Y., BOYD A. J. Microstructure of cement paste subject to early carbonation curing. Cement Concrete Research, n.42, v.1, 2012.

SAMSON, G.; PHELIPOT-MARDEL, A.; LANOS C. A review of thermomechanical of lightweight concrete, vol. 69, 2017.

SHAOEI, P.; AMERI, F.; MUSAEEI, H. R.; GHASEMI, T.; $\mathrm{CHEAH}$, C.B. Glass powder as a partial precursor in Portland cement and alkali-activated slag mortar: A comprehensive comparative study. Construction and Building Materials, 2020.

SIQUEIRA, L. V. M.; STRAMARI, M. R.; FOLGUERAS, M. V. Adição de Poliuretano Expandido para a Confecção de Blocos de Concreto Leve. Revista Matéria, v. 9, n. 4, 2004.

VARGAS, I.M.; WIEBECK, H. Reciclagem de vidro laminado: utilização dos vidros de baixa granulometria como carga abrasiva na formulação de vernizes de alto tráfego para pisos de madeira. Polímeros vol.17. São Paulo, 2007.

VLADIMIROV, V. S.; LUKIN, E. S.; POPOYA, N. A.; ILYUKHIN, A.; MOIZIS, S.E.; ARTAMONOV, M.A. New types of light-weight refractory and heat-insulation materials for long-term use at extremely high temperatures. Glass and Ceramics, v.68, n.3-4, 2011.

YUAN, C., NIU, D., CHEN, N., et al. Influence of carbonation on microstructure of concrete. Guisuanyan Tongbao, vol.32, n.4, 2013.

\section{AGRADECIMENTOS}

Agradecemos a empresa Personal Glass pelo fornecimento do lodo da lapidação de vidro. Este trabalho foi apoiado pela Fundação Amparo para Pesquisa e Inovação do Estado de Santa Catarina [n 06/2017, Grupo de Pesquisa em Materiais Ativos] e teve concessão de Bolsa pelo Programa Institucional de Bolsas de Iniciação Tecnológica e Inovação (PIBITI), do Conselho Nacional de Desenvolvimento Científico e Tecnológico (CNPq).

\section{AUTORES}

ORCID Id: https://orcid.org/0000-0002-4054-4913

POLYANA BAUNGARTEN | Universidade do Sul de Santa Catarina (UNISUL) | e-mail: polyanabaungarten@gmail.com

ORCID iD: https://orcid.org/0000-0001-7306-7984

RACHEL FAVERZANI MAGNAGO, Dra. | Universidade do Sul de Santa Catarina (UNISUL) | e-mail: rachelfaverzanimagnago@gmail.com

\section{COMO CITAR ESTE ARTIGO}

BAUNGARTEN, Polyana; MAGNAGO, Rachel Faverzani. Resistência À Compressão Mecânica de Compósitos de Poliuretano e Cimento, com Adição de Lodo de Vidro como Substituto da Areia. MIX Sustentável, [S.I.], v. 6, n. 2, p. 175-182, mai. 2020. ISSN 24473073. Disponível em:<http://www.nexos.ufsc.br/index.php/ mixsustentavel>. Acesso em: dia mês. ano. doi:https:// doi.org/10.29183/2447-3073.MIX2020.v6.n2.175-182.

DATA DE ENVI0: 18/05/2020

DATA DE ACEITE: 20/05/2020 
